\title{
MRI Volumetric Changes in Hippocampal Subfields in Psychosis: a Protocol for a Systematic Review and Meta-analysis
}

\section{Anurag Nasa}

Department of Psychiatry, Trinity College Institute of Neuroscience https://orcid.org/0000-0002-54989817

\section{Olivia Mosley}

Department of Psychiatry, Trinity College Institute of Neuroscience

\section{Elena Roman}

Department of Psychiatry, Trinity College Institute of Neuroscience

\section{Allison Kelliher}

Department of Psychiatry, Trinity College Institute of Neuroscience

\section{Caoimhe Gaughan}

Department of Psychiatry, Trinity College Institute of Neuroscience

Kirk J. Levins

Department of Anaesthesiology, St Vincents University Hospital

\section{David Coppinger}

Department of Psychiatry, Royal College of Surgeons in Ireland

\section{Erik O’Hanlon}

Department of Psychiatry, Royal College of Surgeons in Ireland

\section{Darren William Roddy ( $\nabla$ dwroddy@tcd.ie )}

Department of Psychiatry, Trinity College Institute of Neuroscience, Lloyd Building, Trinity College Dublin https://orcid.org/0000-0001-7070-5469

Mary Cannon

Department of Psychiatry, Royal College of Surgeons in Ireland

\section{Protocol}

Keywords: Magnetic Resonance Imaging, Hippocampus, Psychosis, Cornu Ammonis, Subiculum, Systematic review, Meta-analysis

Posted Date: January 21st, 2021

DOI: https://doi.org/10.21203/rs.3.rs-150894/v1 
License: (c) (i) This work is licensed under a Creative Commons Attribution 4.0 International License. Read Full License

Version of Record: A version of this preprint was published at Systematic Reviews on March 15th, 2022. See the published version at https://doi.org/10.1186/s13643-022-01916-5. 


\section{Abstract}

Background: The hippocampus has for long been known for its abilities to form new, declarative memory. However, emerging findings across conditions in the psychosis spectrum also implicate its role in emotional regulation. Systematic reviews have demonstrated consistent volume atrophic changes in the hippocampus. The aim of the systematic review and metanalysis which will follow from this protocol will be to investigate the volume-based neuroimaging findings across each of the subfields of the hippocampus in psychosis independent of diagnosis.

Methods: Volume changes across subfields of the hippocampus in psychotic illnesses will be assessed by systematic review following the Preferred Reporting Items for Systematic Reviews and Meta-Analyses (PRISMA). MRI neuroimaging studies of patients with a definitive diagnosis of psychosis (including brief pre diagnostic states) will be included. Studies lacking adequate controls, illicit drug use, medical psychosis, history of other significant psychiatric comorbidities or emphasis on age groups above 65 or below 16 will be excluded. Subfields investigated will include the CA1, CA2/3, CA4, subiculum, presubiculum, dentate gyrus, stratum, molecular layer, granular cell layer, entorhinal cortex and fimbria. Two researchers will independently conduct the search and selection procedures to gather suitable studies. One reviewer will independently perform all the data extraction, and another reviewer will then systemically check all the extracted information using the original articles to ensure accuracy. Statistical analysis will be performed using the metafor and meta packages in R Studio with application of the random effects model.

Discussion: This study will provide insight into the volumetric changes in psychosis of the subfields of the hippocampus, independent of diagnosis. This may shed light on the intricate neural pathology which encompasses psychosis and will open avenues for further exploration of the structures identified as potential drivers of volume change.

Systematic review registration: PROSPERO CRD42020199558

\section{Background}

\section{Psychosis}

Psychosis is a broad term that encompasses symptoms related to a change in perception of reality. It is found in many psychiatric, neurologic, neurodevelopmental and medical conditions [1]. The diseases in which psychosis is considered a core symptom are outlined in the Schizophrenia Spectrum and Other Psychotic Disorders section of the Diagnostic and Statistical Manual of Mental Disorders: Fifth edition (DSM-5) [2]. Such disorders include: schizotypal (personality) disorder, delusional disorder, brief psychotic disorder, schizophreniform disorder, schizophrenia, schizoaffective disorder, substance/medicationinduced psychotic disorder and psychotic disorder due to another medical condition and various catatonias [2]. DSM-5 states that these disorders "are defined by abnormalities in one or more of the following five domains: delusions, hallucinations, disorganized thinking (speech), grossly disorganized or 
abnormal motor behaviour (including catatonia), and negative symptoms." [1]. Furthermore, psychosis as a defined by these abnormalities is also found as a qualifier in other non-primarily psychotic conditions, including Major Depressive Disorder, Bipolar Affective Disorder and Post Traumatic Stress Disorder. Schizophrenia is considered the archetypal psychotic disorder with an incidence of 15.2 per 100,000 (McGrath). However, psychotic symptoms are not confined to schizophrenia and 1.5 to $3.5 \%$ of people will meet diagnostic criteria for a psychotic disorder at some point in their lifetime (Van os). Many brain areas have implicated in psychosis with the hippocampus being one of the regions most consistently identified in studies.

\section{Hippocampus and substructures}

The hippocampus, located deep within the medial temporal lobe, is one of the most investigated structures in the brain [3]. Initial research concentrated on its role in forming new, declarative memories [4, 5] and spatial organisation [6], however, as findings emerged of hippocampal involvement across psychiatric disorders, the hippocampal role in emotional regulation became a focus for research [7-11]. The hippocampus is structurally plastic throughout life and is one of the few areas that can undergo adult neurogenesis in the brain [12]. As an extension underneath the cerebral cortex it lies along the floor of the lateral ventricle, and forms an integral hub of the limbic system [13]. The hippocampus has an Sshaped structure consists of two histologically distinct parts separated by the hippocampal fissure: the hippocampus proper or cornu ammonis (CA) region and the dentate gyrus [13]. The CA regions lie on the subiculum, which extends to the entorhinal cortex. The $\mathrm{CA}$, dentate and subiculum together form a unit that is often referred to as the hippocampal formation or 'hippocampus' [8]. A unique feature of the structure is the largely (but not exclusively) unidirectional information flow through the hippocampal system [14]. External sensory and internal cortical/subcortical information funnels through the entorhinal cortex to enter the hippocampus via the dentate granule cells. The dentate can be considered the preprocessor of inputs into the hippocampus [15], playing a critical role in mediating some of the higher brain functions of the hippocampus, namely memory, learning and spatial coding [16]. It is also the only area within the hippocampus where neurogenesis occurs [17], allowing for new neurons to be generated throughout life. Dentate neurogenesis dysfunction has been implicated in some psychiatric disorders [18-20]. From the dentate, information flows to the CA regions. The CA region is divided into four regions (CA1-3), with CA4 lying within the hilum of the dentate and considered functionally part of this structure [21]. The dentate to the CA3 pathway is known as the mossy fiber pathway and the CA3 region has been implicated in spatial awareness [6] and as a hippocampal pacemaker coordinating alertness and encoding [22]. Aberrant CA3 neuronal activity has been shown in schizophrenia [23]. CA2 is a relatively small and indistinct area interposed between the larger CA3 and CA1 and appears to have unique connectivity with the amygdala and HPA axis [24] and has suggested role in social cognition [25]. Smaller CA2 regions have been found in post-mortem brains of schizophrenia patients [26]. CA1 is by far the largest hippocampal subfield [27] and has a role in autobiographical memory [28] with pathology being shown in dementia [29]. Finally, information flows from from the CA regions to the subiculum. The human subicular region is divided into the subiculum, presubiculum and parasubiculum [30]. The pre and parasubiculum are more parahippocampal in origin and function and may be considered input hubs of 
the entorhinal cortex [31]. With the CA1 region, the subiculum proper may be considered the output region of the hippocampus, with both structures returning information to the deep entorhinal cortex and directly out from the hippocampus.

\section{Hippocampal MRI and psychosis}

Hippocampal involvement across the psychosis spectrum has been demonstrated with smaller hippocampal volumes consistently shown in psychotic disorders such as schizophrenia [32] and schizoaffective disorder [33]. Patient with bipolar affective disorder with psychosis appear to show smaller hippocampi [34] but not bipolar patients without psychosis [35]. Conversely, although depression is consistently associated with smaller hippocampal volumes [7], depression with psychosis appears to show little association with hippocampal volume [36]. 'Premorbid' and 'at-risk' psychotic states where individuals experience brief or limited psychotic symptoms (and often go on to develop true psychotic conditions) are also associated with smaller hippocampal volumes [10].

Recent hardware advances MRI such as increased fields strengths ( $3 \mathrm{~T}, 4 \mathrm{~T}, 7 \mathrm{~T}$, and higher), improved acquisition protocols and the development of sophisticated pre-processing techniques combined with improved computational power have allowed greater accuracy and speed in quantifying hippocampal volumes. Specifically, the increased resolution achieved through these advances has allowed researchers to consistently quantify hippocampal volumes at the substructure level (e.g. CA1-4, subiculum, dentate etc above). The advent of automated segmentation techniques based on detailed high-resolution atlases has facilitated the measurement of hippocampal subfields in larger datasets. All hippocampal regions have been shown by various studies to be smaller in psychotic disorders. Although there have been disorder specific reviews of hippocampal subfields in schizophrenia [37] and bipolar disorder [38], a comprehensive review and meta-analysis of hippocampal subfields focusing on the presence of psychosis rather than specific diagnoses has yet to be published. A common hippocampal subfield signature across the entire psychotic spectrum may provide deeper insights into the aetiology of the symptoms of psychosis and potentially reveal new common therapeutic targets for psychosis. Hence, the aim of the systematic review and metanalysis which will follow from this protocol will be to investigate the volume-based neuroimaging findings across each of the subfields of the hippocampus in psychosis independent of diagnosis.

\section{Methods}

\section{Search strategy}

Online databases will be searched for the relevant articles. The databases examined will include PubMed, Google Scholar, MEDLINE and EMBASE, from where articles will be systematically assessed to identify those relevant to our hypothesis. The search performed was as follows: (Hippocampus AND psychosis) OR (Hippocampal subfield AND psychosis) OR (Cornu ammonis AND psychosis) OR (Subiculum AND psychosis) OR (dentate AND psychosis) OR (Hippocampus AND Brief Limited Intermittent Psychotic Symptoms) OR (Hippocampal Subfield AND Brief Limited Intermittent Psychotic Symptoms) OR 
(Hippocampus AND Schizophrenia) OR (Hippocampus AND Bipolar Disorder) OR (Hippocampus AND Depression) OR (Hippocampus AND Schizoaffective Disorder) OR (Hippocampus AND Delusional Disorder) OR (Hippocampus AND Brief Psychotic Disorder) OR (Hippocampus AND Schizophreniform Disorder) OR (Hippocampus AND Medication Induced Psychotic Disorder) OR (Hippocampus AND Hallucinations) OR (Hippocampus AND Delusions) OR (Hippocampus AND Thought Disorder) OR (Hippocampus AND Catatonia) OR (Hippocampus AND Personality Disorder)

References from the output articles will also be checked, and articles which are pertinent to our study will also be incorporated into it. The search items will be rerun before publication to include newer studies which got added to the databases.

\section{Eligibility Selection}

The studies to be included in this review will encompass MRI neuroimaging studies of patients with a definitive diagnosis of psychosis, and comparisons with healthy control participants. The studies which segmented the subfields of the hippocampus using either automatic or manual means will be included. Studies lacking a control group will be excluded. All the studies included will have been peer-reviewed. Although the search items were in English, we will include non-English studies, which we'll get translated professionally, and contact the corresponding author with any confusions which may arise. Studies where illicit drug use is documented, or those with history of other significant psychiatric comorbidities will be excluded. Studies with participants that had medical psychosis will also be excluded since psychiatric psychosis is the focus of this review. Studies with an emphasis on ages above 65 or below 16 will also be excluded. Incomplete or ambiguous information will be clarified by contacting the corresponding authors of the respective studies. In instances where the results from a study has been reported in more than one article, the results from the article with greater sample size will be extracted.

Two researchers will independently conduct the search and selection procedures to gather suitable studies. We will include case-control, cohort, cross-sectional studies, randomised control trials and longitudinal studies. Inconsistencies regarding the studies gathered will be discussed and resolved with guidance from Dr Roddy.

\section{Data Collection}

One reviewer will independently perform all the data extraction on an excel spreadsheet, and another reviewer will then systemically check all the extracted information using the original articles to ensure accuracy. The information to be extracted includes the following:

1. Author and publication year

2. Cohort information (the specific psychotic illness(es) the study discusses)

3. Sample and descriptive demographic information (age, gender)

4. Age of onset, duration of illness, duration of untreated psychosis 
5. Diagnostic method and quantification criteria for psychosis with results e.g. PANSS, BPRS, SAPS + SANS

6. Whether the participants were medicated or not. If so, which medication class was used?

7. Software used for hippocampal subfield volume determination

8. Type of MRI scanner used, and magnetic field strength

9. Volumetric information for each of the subfields in both hemispheres

10. Illicit drug use

\section{Hippocampal Subfield Volumes}

Quite often in neuroimaging studies, the definition and segmentation of subfields is conducted using differing methodologies and softwares: manual or automatic. Though the output from these should be very similarly, though we will be cautious of possible heterogeneity which may arise due to this by recording the software used in each instance, allowing for a possible explanation of heterogeneity if it arises during our analysis.

The subfield volume information we will extract will be that of the patients and controls within each of the studies. We will be extract volumes of the CA1, CA2, CA3, DG, CA4, Dentate Gyrus (DG), Subiculum, Presubiculum. In instances where composite volumes are presented in the paper, those will be recorded. CA4-DG and CA2-3 are pairs which can be difficult to tell differentiate, particularly using the automated softwares. Hence, we expect that we'll frequently be recording composite volumes for those and will be running analysis on them as a composite.

\section{Meta-analysis}

Statistical analysis will be conducted using the metaphor and meta packages in R Studio 2020 (RStudio, PBC, Boston, MA; URL http://www.rstudio.com), which is an integrated development environment for $R$ $[39,40]$. Given the assumption of exchangeability in a random-effects model, it will be applied throughout our review to weight each study and control for potential heterogeneity [41, 42]. Potential heterogeneity we may encounter has been explored and includes the software used for subfield segmentation, duration of illness, type of psychotic disorder, age of onset, medication use, measurement on scales for psychosis and magnetic field strength of the MRI scanner used. Cohen's d statistic or Hedges' unbiased g will be computed, as appropriate, for an effect size of the difference between means of the patient and control groups[43]. Potential type 1 inflation errors will be addressed using conservative correction measures such as false discovery rate [44] or Bonferroni [45].

\section{Meta-regression}

Meta-regression will be employed for assessment of secondary and tertiary hypotheses that subfield volumes change with psychosis scores and with duration of illness. An analysis will be performed to examine the relationship between standardized mean differences (SMD) of the measurements on psychosis scales and Cohen's $d$ for volume changes in each of the subfields. Another analysis will 
examine the relationship between duration of illness and volume changes in each of the subfields. Regression will be performed using SPSS-26 (IBM SPSS Statistics 26 for Windows 10).

\section{Between-study heterogeneity}

Assessment of between-study heterogeneity will be conducted using the Cochran's Q, and the degree of heterogeneity will be quantified using the $\mathrm{I}^{2}$ statistic [46]. This will give us the percentage of variability that is due to differences between studies compared to sampling variance. The interpretation of these ${ }^{2}$ values will be $0.25=$ low, 0.5 moderate, $0.75=$ high. The significance threshold for establishing the studies are heterogenous will be 0.1 . In instances where the Q-statistic is significant, Galbriath plots will be produced to supplement forest plots in determining the studies which have the largest influence on increasing the heterogeneity [47].

\section{Bias}

Quality of evidence will be evaluated using the Grading of Recommendations, Assessment, Development and Evaluations (GRADE) criteria with five domains of evidence being assessed (risk of bias, imprecision, inconsistency, indirectness, and publication bias) each according to four levels of quality (very low, low, moderate, and high) [48]. Publication bias and small-study effects will be important features addressed as part of this review. These occur when mostly the significant findings get published [49]. Small study bias refers to phenomena where studies with smaller samples, and less power, tend to report larger effect sizes [50]. These will be verified by visual inspection of funnel plots and assessed using Eggers test [51]. Studies which don't appear adequately robust will be eliminated from the quantitative meta-analysis but may be documented narratively throughout the paper. Risk of bias (i.e. flaws in study design, conduct or analysis) will be assessed using Newcastle-Ottawa Scale, where studies will be graded according to three quality outcomes: group selection, group comparability, and outcome [52].

\section{Data Synthesis}

This study will obtain clinical, demographic, and methodological variants. A forest plot will be used to synthesise the total number of participants, studies, subfield volumes with mean differences, 95\% confidence intervals, $\mathrm{p}$ values, and $\mathrm{I}^{2}$ statistics in graphical form [53]. If a meta-analytical approach is not feasible based on heterogeneity and sample sizes, we plan to summarise the findings as a narrative systematic review.

\section{Conclusion}

This is a protocol for a systematic review and meta-analysis with the objective of summarizing findings from MRI neuroimaging studies of the hippocampal subfields in psychosis. While hippocampal atrophy has been well documented in prior studies, this study will provide another layer of specificity in outlining the subfields which drive that effect. Adjacent to this, it will seek whether duration of illness and degree of psychosis impact these effects. 


\section{Declarations}

\section{ETHICAL APPROVAL AND CONSENT TO PARTICIPATE}

Not applicable

\section{CONSENT FOR PUBLICATION}

Not applicable

\section{AVAILABILITY OF DATA AND MATERIAL}

Data sharing is not applicable to this article as no datasets were generated or analysed during the current study.

\section{COMPETING INTERESTS}

The authors declare they have no competing interests

\section{FUNDING}

This project was funded through the ERC as part of iHEAR project

\section{AUTHOR CONTRIBUTIONS}

AN: Writing, editing, formatting manuscript, statistics

OM: Writing, proofreading and editing

ER: Writing, proofreading and editing

AK: Proofreading and editing

CG: Proofreading and editing

KJL: Proofreading and editing, feasibility review

DC: Proofreading and editing

EOH: Proofreading and editing, statistics, lead investigator 
DWR: Proofreading and editing, formatting manuscript, lead investigator, study design

MC: Proofreading and editing, guarantor, lead investigator

\section{ACKNOWLEDGEMENTS}

Not applicable

\section{References}

1. Schrimpf LA, Aggarwal A, Lauriello J: Psychosis. CONTINUUM: Lifelong Learning in Neurology 2018, 24:845-860.

2. Association AP: Diagnostic and statistical manual of mental disorders (DSM-5®). American Psychiatric Pub; 2013.

3. Duvernoy HM: The human hippocampus : functional anatomy, vascularization, and serial sections with MRI. 3rd ed. edn. Berlin Berlin ; New York: Springer; 2005.

4. Francis AN, Seidman LJ, Tandon N, Shenton ME, Thermenos HW, Mesholam-Gately RI, van Elst LT, Tuschen-Caffier B, DeLisi LE, Keshavan MS: Reduced subicular subdivisions of the hippocampal formation and verbal declarative memory impairments in young relatives at risk for schizophrenia. Schizophrenia research 2013, 151:154-157.

5. O'Keefe J, Nadel L: The hippocampus as a cognitive map. Oxford: Clarendon Press; 1978.

6. O'Keefe J, Dostrovsky J: The hippocampus as a spatial map. Preliminary evidence from unit activity in the freely-moving rat. Brain Res 1971, 34:171-175.

7. Roddy DW, Farrell C, Doolin K, Roman E, Tozzi L, Frodl T, O'Keane V, O'Hanlon E: The Hippocampus in Depression: More Than the Sum of Its Parts? Advanced Hippocampal Substructure Segmentation in Depression. Biological Psychiatry 2018.

8. Roddy D, O'Keane V: Cornu Ammonis Changes Are at the Core of Hippocampal Pathology in Depression. Chronic Stress 2019, 3:2470547019849376.

9. Nolan M, Roman E, Nasa A, Levins KJ, O'Hanlon E, O'Keane V, Willian Roddy D: Hippocampal and Amygdalar Volume Changes in Major Depressive Disorder: A Targeted Review and Focus on Stress. Chronic Stress 2020, 4:2470547020944553.

10. Calvo A, Roddy DW, Coughlan H, Kelleher I, Healy C, Harley M, Clarke M, Leemans A, Frodl T, O'Hanlon $\mathrm{E}$ : Reduced hippocampal volume in adolescents with psychotic experiences: A longitudinal population-based study. Plos one 2020, 15:e0233670.

11. Chen LW, Sun D, Davis SL, Haswell CC, Dennis EL, Swanson CA, Whelan CD, Gutman B, Jahanshad N, Iglesias JE, et al: Smaller hippocampal CA1 subfield volume in posttraumatic stress disorder. Depress Anxiety 2018, 35:1018-1029. 
12. Leuner B, Gould E: Structural plasticity and hippocampal function. Annual review of psychology 2010, 61:111-140.

13. Anand KS, Dhikav V: Hippocampus in health and disease: An overview. Annals of Indian Academy of Neurology 2012, 15:239.

14. Amaral DG, Scharfman HE, Lavenex P: The dentate gyrus: fundamental neuroanatomical organization (dentate gyrus for dummies). Progress in brain research 2007, 163:3-790.

15. Jonas P, Lisman J: Structure, function, and plasticity of hippocampal dentate gyrus microcircuits. Frontiers in neural circuits 2014, 8:107.

16. Aimone JB, Deng W, Gage FH: Resolving new memories: a critical look at the dentate gyrus, adult neurogenesis, and pattern separation. Neuron 2011, 70:589-596.

17. Abrous DN, Koehl M, Le Moal M: Adult neurogenesis: from precursors to network and physiology. Physiol Rev 2005, 85:523-569.

18. Boldrini M, Underwood MD, Hen R, Rosoklija GB, Dwork AJ, John Mann J, Arango V: Antidepressants increase neural progenitor cells in the human hippocampus. Neuropsychopharmacology 2009, 34:2376-2389.

19. Sahay A, Drew MR, Hen R: Dentate gyrus neurogenesis and depression. Progress in brain research 2007, 163:697-822.

20. Samuels BA, Hen R: Neurogenesis and affective disorders. Eur J Neurosci 2011, 33:1152-1159.

21. Amaral DG: A Golgi study of cell types in the hilar region of the hippocampus in the rat. $J$ Comp Neuro/ 1978, 182:851-914.

22. Wilson MA, McNaughton BL: Reactivation of hippocampal ensemble memories during sleep. Science 1994, 265:676-679.

23. Behrendt R-P: Contribution of hippocampal region CA3 to consciousness and schizophrenic hallucinations. Neuroscience \& Biobehavioral Reviews 2010, 34:1121-1136.

24. Pikkarainen M, Rönkkö S, Savander V, Insausti R, Pitkänen A: Projections from the lateral, basal, and accessory basal nuclei of the amygdala to the hippocampal formation in rat. Journal of Comparative Neurology 1999, 403:229-260.

25. Hitti FL, Siegelbaum SA: The hippocampal CA2 region is essential for social memory. Nature 2014, 508:88.

26. Narr KL, Thompson PM, Szeszko P, Robinson D, Jang S, Woods RP, Kim S, Hayashi KM, Asunction D, Toga AW: Regional specificity of hippocampal volume reductions in firstepisode schizophrenia. Neuroimage 2004, 21:1563-1575.

27. West MJ, Coleman PD, Flood DG, Troncoso JC: Differences in the pattern of hippocampal neuronal loss in normal ageing and Alzheimer's disease. The Lancet 1994, 344:769-772.

28. Kohler CA, Carvalho AF, Alves GS, Mclntyre RS, Hyphantis TN, Cammarota M: Autobiographical Memory Disturbances in Depression: A Novel Therapeutic Target? Neural Plast 2015, 2015:759139. 
29. Mueller S, Stables L, Du A, Schuff N, Truran D, Cashdollar N, Weiner M: Measurement of hippocampal subfields and age-related changes with high resolution MRI at 4 T. Neurobiology of aging 2007, 28:719-726.

30. Witter MP, Groenewegen H, Da Silva FL, Lohman A: Functional organization of the extrinsic and intrinsic circuitry of the parahippocampal region. Progress in neurobiology 1989, 33:161-253.

31. Witter MP, Groenewegen HJ: The subiculum: cytoarchitectonically a simple structure, but hodologically complex. In Progress in brain research. Volume 83: Elsevier; 1990: 47-58

32. Wright IC, Rabe-Hesketh S, Woodruff PW, David AS, Murray RM, Bullmore ET: Meta-analysis of regional brain volumes in schizophrenia. American Journal of Psychiatry 2000, 157:16-25.

33. Arnold SJ, Ivleva El, Gopal TA, Reddy AP, Jeon-Slaughter H, Sacco CB, Francis AN, Tandon N, Bidesi AS, Witte B: Hippocampal volume is reduced in schizophrenia and schizoaffective disorder but not in psychotic bipolar I disorder demonstrated by both manual tracing and automated parcellation (FreeSurfer). Schizophrenia bulletin 2015, 41:233-249.

34. Strasser HC, Lilyestrom J, Ashby ER, Honeycutt NA, Schretlen DJ, Pulver AE, Hopkins RO, Depaulo JR, Potash JB, Schweizer B: Hippocampal and ventricular volumes in psychotic and nonpsychotic bipolar patients compared with schizophrenia patients and community control subjects: a pilot study. Biological psychiatry 2005, 57:633-639.

35. Mathew I, Gardin TM, Tandon N, Eack S, Francis AN, Seidman LJ, Clementz B, Pearlson GD, Sweeney JA, Tamminga CA: Medial temporal lobe structures and hippocampal subfields in psychotic disorders: findings from the Bipolar-Schizophrenia Network on Intermediate Phenotypes (B-SNIP) study. JAMA psychiatry 2014, 71:769-777.

36. Keller J, Shen L, Gomez RG, Garrett A, Solvason HB, Reiss A, Schatzberg AF: Hippocampal and amygdalar volumes in psychotic and nonpsychotic unipolar depression. American Journal of Psychiatry 2008, 165:872-880.

37. Hu N, Luo C, Zhang W, Yang X, Xiao Y, Sweeney JA, Lui S, Gong Q: Hippocampal subfield alterations in schizophrenia: A selective review of structural MRI studies. Biomarkers in Neuropsychiatry 2020:100026.

38. Haukvik UK, Tamnes CK, Söderman E, Agartz I: Neuroimaging hippocampal subfields in schizophrenia and bipolar disorder: a systematic review and meta-analysis. Journal of Psychiatric Research 2018, 104:217-226.

39. Viechtbauer W: Conducting meta-analyses in R with the metafor package. Journal of statistical software 2010, 36:1-48.

40. Team R: RStudio: Integrated development for R. RStudio Inc.(2015). Acknowledgements This work was supported by Fundação para a Ciência e Tecnologia (FCT), Azores 2020.

41. DerSimonian R, Laird N: Meta-analysis in clinical trials. Controlled clinical trials 1986, 7:177-188.

42. Riley RD, Higgins JP, Deeks JJ: Interpretation of random effects meta-analyses. Bmj $2011,342$.

43. Grissom RJ: Difference between Cohen's d and Hedges'g for effect size metrics. 
44. Benjamini Y, Hochberg Y: Controlling the false discovery rate: a practical and powerful approach to multiple testing. Journal of the Royal statistical society: series B (Methodological) 1995, 57:289-300.

45. Sedgwick P: Multiple hypothesis testing and Bonferroni's correction. Bmj 2014, 349:g6284.

46. Higgins JP, Thompson SG, Deeks JJ, Altman DG: Measuring inconsistency in meta-analyses. Bmj 2003, 327:557-560.

47. Galbraith RF: The radial plot: graphical assessment of spread in ages. International Journal of Radiation Applications and Instrumentation Part D Nuclear Tracks and Radiation Measurements 1990, 17:207-214.

48. Guyatt GH, Oxman AD, Vist GE, Kunz R, Falck-Ytter Y, Alonso-Coello P, Schünemann HJ: GRADE: an emerging consensus on rating quality of evidence and strength of recommendations. Bmj 2008, 336:924-926.

49. Easterbrook PJ, Gopalan R, Berlin J, Matthews DR: Publication bias in clinical research. The Lancet 1991, 337:867-872.

50. Thorlund K, Imberger G, Walsh M, Chu R, Gluud C, Wetterslev J, Guyatt G, Devereaux PJ, Thabane L: The number of patients and events required to limit the risk of overestimation of intervention effects in meta-analysis-a simulation study. PloS one 2011, 6:e25491.

51. Egger M, Smith GD, Schneider M, Minder C: Bias in meta-analysis detected by a simple, graphical test. Bmj 1997, 315:629-634.

52. Lo CK-L, Mertz D, Loeb M: Newcastle-Ottawa Scale: comparing reviewers' to authors' assessments. BMC medical research methodology 2014, 14:45.

53. Lewis S, Clarke M: Forest plots: trying to see the wood and the trees. Bmj 2001, 322:1479-1480.

\section{Supplementary Files}

This is a list of supplementary files associated with this preprint. Click to download.

- PRISMAPchecklist.docx 